\title{
RÉPRESSION PARTICIPATIVE ET CONTRÔLE SOCIAL DE LA DISSIDENCE SOUS L'AKP EN TURQUIE
}

\section{Cléa Pineau}

\author{
ARPoS | «Pôle Sud » \\ 2020/2 n 53 | pages 53 à 68 \\ ISSN 1262-1676 \\ ISBN 9782918036319 \\ DOI 10.3917/psud.053.0053
}

\section{Article disponible en ligne à l'adresse :}

https://www.cairn.info/revue-pole-sud-2020-2-page-53.htm

Distribution électronique Cairn.info pour ARPoS.

(C) ARPoS. Tous droits réservés pour tous pays.

La reproduction ou représentation de cet article, notamment par photocopie, n'est autorisée que dans les limites des conditions générales d'utilisation du site ou, le cas échéant, des conditions générales de la licence souscrite par votre établissement. Toute autre reproduction ou représentation, en tout ou partie, sous quelque forme et de quelque manière que ce soit, est interdite sauf accord préalable et écrit de l'éditeur, en dehors des cas prévus par la législation en vigueur en France. Il est précisé que son stockage dans une base de données est également interdit. 


\title{
RÉPRESSION PARTICIPATIVE ET CONTRÔLE SOCIAL DE LA DISSIDENCE SOUS L'AKP EN TURQUIE
}

\author{
Cléa Pineau \\ Centre Européen de Sociologie et de Science Politique \\ Université Paris 1 Panthéon-Sorbonne \\ pineau.clea@gmail.com
}

\section{RÉSUMÉ/ABSTRACT}

Par opposition à la thèse gramscienne, cette contribution montre que la répression n'est pas antinomique au renforcement de la légitimité d'un État. Elle étudie, à partir du cas de la Turquie contemporaine, comment un parti politique au sommet de l'État, le Parti de la Justice et du Développement (AKP), peut mettre en place des mécanismes répressifs, auxquels la population civile participe, qui consolident sa légitimité. Les ressources sont distribuées et les conduites sont récompensées ou réprimées selon leur conformité à une nouvelle forme de "citoyenneté " ("vatandaşlık») définie selon des logiques partisanes. Ces mécanismes permettent à l'ÉtatAKP de produire des subjectivés dominées et actives dans l'entretien des dispositifs exclusifs et punitifs. Les pratiques coercitives sont encouragées par l'État-AKP et utilisées pour contrôler les conduites dissidentes au sein de la société. L'encouragement de la répression participative est légitimé par le discours sécuritaire faisant appel au registre de la « menace terroriste » et renforcé par l'informatisation des techniques de pouvoir.

In contrast to the Gramscian thesis, this contribution shows that repression is not antinomic with the reinforcement of state legitimacy. It examines, based on the case of contemporary Turkey, how a political party which control the state, the Justice and Development Party (AKP), can produce repressive mechanisms, in which the civilian population participates, that consolidate its legitimacy. Resources are distributed and behaviors are rewarded or repressed according to their conformity with a new form of 'citizenship' ("vatandasllik") defined through partisan logics. These mechanisms allow the AKP-state to produce subjectivities that are both dominated and active in the maintenance of exclusionary and punitive dispositifs. Coercive practices are encouraged by the AKP-state and used to control dissenting conducts within society. Using the register of the "terrorist threat", the encouragement of participatory repression is legitimised and reinforced by the digitalisation of technologies of power.

\section{MOTS-CLÉS/KEYWORDS}

Turquie, AKP, autoritarisme, terrorisme, répression, subjectivation, domination Turkey, AKP, authoritarianism, terrorism, repression, subjectification, domination 
"S’il te plaît, parle-moi d'autre chose maintenant », demanda Ka avec délicatesse. İpek racontait l'arrestation sur dénonciation de quelqu'un qui passait par les jardins intérieurs, à deux immeubles de là ; elle s'arrêta, pleine de compassion.

Ka vit de la peur dans ses yeux. "

(Extrait de Neige, de O. Pamuk, traduit par J.-F. Pérouse, 2005 [2002], p. 345)

\section{INTRODUCTION}

Contrairement à la thèse gramscienne, la répression n'est pas antinomique au renforcement de la légitimité étatique et les répertoires d'action répressifs de l'État en contexte autoritaire ne se limitent pas aux dispositifs traditionnellement étudiés tels que l'armée et la police. Une catégorie d'acteurs non étatiques peut participer à l'entretien et au renforcement des dispositifs répressifs : la population civile (Hibou, 2005). Cette participation de la population à la répression peut être analysée comme le résultat d'une "opération " au sens foucaldien. Au sein de cette " opération " sont installés des mécanismes de normalisation disciplinaire qui reposent sur un modèle prédéfini et « consiste[nt] à essayer de rendre les gens, les gestes, les actes conformes à ce modèle, le normal étant précisément ce qui est capable de se conformer à cette norme et l'anormal, ce qui n'en est pas capable " (Foucault, 2004, p. 59). Dans cette contribution, janalyse ces mécanismes comme des processus de subjectivation au travers de la définition d'une communauté citoyenne partisane excluant tout individu et comportement dissident vis-à-vis du modèle développé par un parti politique qui a investi l'État. La participation des citoyens à la défense du modèle via le développement de techniques de soi et de pratiques de domination envers les autres contribue à renforcer la légitimité étatique (Bayart, 2008). L'analyse repose sur l'étude de la gouvernementalité du parti de la Justice et du Développement (AKP - Adalet ve Kalkınma Partisi) en Turquie de 2002 à aujourd'hui. L'État turc peut être envisagé comme un État-parti dans le sens où l'AKP a investi l'État en y développant son réseau partisan, contrôle un grand nombre de champs (économique, politique, médiatique, religieux) et s’attèle à la destruction de toute forme d'opposition (Dorronsoro, 2017). De plus, les techniques de pouvoir développées par l'État-AKP mettent en concurrence les individus pour l'accès aux ressources sur la base de leur soutien au parti (Ark, 2015).

Le développement de l'appareil coercitif sous l'AKP s'insère dans le contexte global d'une rationalisation du discours sécuritaire et d'une informatisation des techniques de gouvernement présentée sous forme de progrès social. En Turquie, ce progrès social est notamment concrétisé à partir du projet de la "smart city " ("Akillı Şehir »). À l'occasion du congrès sur " les villes et municipalités intelligentes » qui s'est déroulé à Ankara en janvier 2020, le président de la République, Recep Tayyip Erdoğan, insiste sur sa volonté d'apporter une amélioration des services et une augmentation de la qualité de vie des citoyens. Mustafa Varank, ministre de l'Industrie et des Technologies, rappelle quant à lui la nécessité de 
gérer les "nouvelles " menaces urbaines générées par l'exode rural ${ }^{2}$. La mise en application légale de la "smart city" a permis à la police nationale d'inaugurer dès 2005 un outil de sécurité urbaine par vidéosurveillance à partir du système MOBESE (MOBil Elektronik Sistem Entegrasyonu, Mobile Electronic System Integration) dans les villes d'Istanbul, Ankara, Mersin et Diyarbakır qui a vocation à être implémenté dans l'ensemble des villes du pays (Güler, Kula, 2016, p. 248). Ce programme a pour but de prévenir et de combattre le crime afin d'offrir un environnement sécurisé propice à la consommation et au tourisme (Lyon, 2001 cité par Güler, Kula, op. cit., p. 250) dans des métropoles perçues comme sujettes à l'installation de migrants - qui seraient amenés à s'engager dans des activités criminelles pour pallier à leurs difficultés d'intégration (Özden 2008 cité par Güler, Kula, op. cit, p. 250). Toujours dans le cadre de la "ville intelligente", des living labs (laboratoires vivants) seront développés par les mairies, comme dans le cas pilote de la mairie AKP de Başakşehir à Istanbul étudié par Ceren Ark (2015 op. cit.; Ark, Smyrl 2017). Légitimés par les instances européennes et présentés au travers d'un discours axé sur la "modernité ", les living labs sont utilisés à des fins politiques et justifient notamment «l'installation obligatoire d'une infrastructure informatique dans tous les nouveaux logements » (ibid. p .97).

La ville est un cadre d'analyse pertinent en Turquie, de par les enjeux qui lui sont liés mais aussi du fait de son poids démographique et politique. En Turquie, la population urbaine du pays s'élève à $77 \%$ de la population totale en $2013^{3}$ et les réformes administratives entreprises au cours de la période AKP participent à élargir le poids des métropoles au détriment des espaces ruraux peu à peu radiés du paysage étatique (Pérouse, 2012). Le projet de l'AKP vise à produire une société urbaine disciplinée et homogène dans un pays où les appartenances ethniques et religieuses sont variées et politisées (Débarre, Pérouse, 2016). Lurbain est le lieu d'investissement d'une mission " civilisatrice » ${ }^{4}$ basée sur la promotion de l'islam sunnite et d'une société patriarcale, gérontocratique et consumériste (Pérouse, 2017).

Comment l'État-AKP produit-il une société disciplinée capable de contrôler les conduites dissidentes en son sein ? J'émets l'hypothèse qu'il s'appuie sur le registre sécuritaire afin de générer des mécanismes disciplinaires. Dans un premier temps, j'explique les modes de production d'une société disciplinée et partisane à travers l'ordonnancement de lieux et de conduites correspondant au modèle AKP. J'observe comment cette production d'une communauté citoyenne partisane s'opère à partir d'une répression par l'expulsion punitive de toute forme dissidente. Le registre sécuritaire, élaboré autour de la redéfinition de la catégorie

1. Pour une réflexion approfondie sur la construction sociale de «nouvelles» menaces et du discours sécuritaire associé dans des sociétés où les migrations sont perçues comme telles voir Didier Bigot (1998, 2001).

2. Information disponible sur le site officiel du congrès : http://smartcities.tbb.gov.tr/Smart_Cities_ and_Muncipalities_Congress_and_Exhibition_has_started.html.

3. TÜİK, «Adrese Dayalı Nüfus Kayıt Sistemi Sonuçları - 2013 », Haber Bülteni, no 15974, 29 janvier 2014. http://www.tuik.gov.tr/PdfGetir.do?id=15974 [Consulté le 15 mai 2017].

4. Cette " mission " s'inscrit " dans la continuité discursive du projet modernisateur, au fondement de la République, et TOKI est bien un agent de l'État auto-investi d'une mission civilisatrice " (Pérouse, 2013, p.184.) 
du terrorisme, redéfinit la figure de "l'ennemi ». La menace, diffuse, permet à l'État-parti de gouverner les populations dans un climat d'incertitude favorable au renforcement de sa légitimité et au développement de mécanismes coercitifs. Dans un second temps, je remarque quau-delà de produire des subjectivités en accord avec le modèle, l'État-parti entreprend et tolère une " décharge ", au sens développé par Béatrice Hibou (1999), de son monopole de la violence légitime. La population civile peut être active dans l'exercice de la coercition afin de défendre le modèle prôné par l'AKP. Les formes de lynchages et de délation sont des pratiques encouragées ou tolérées par l'État-AKP comme une modalité de contrôle social et d'usage des conflits sociaux au profit du parti.

Lanalyse repose sur des enquêtes de terrain dans la ville de Diyarbakır de février à mai 2015 et à Mersin de juillet 2016 à mars 2019 sur une période de huit mois ${ }^{5}$. À Diyarbakır, une vingtaine d'entretiens semi-directifs ont été réalisés auprès de consommateurs d'électricité, responsables et employés ou anciens employés de l'entreprise de distribution d'électricité ainsi que d'élus de la mairie dirigée par le parti de la paix et de la démocratie (BDP - Barış ve Demokrasi Partisi) ${ }^{6}$. À Mersin, dans le cadre d'une recherche doctorale, quatre-vingt-dix entretiens semi-directifs et des entretiens informels ont été effectués majoritairement auprès de militants des principaux partis d'oppositions, associations, chambres professionnelles, syndicats, employés et cadres de mairies d'opposition, hommes d'affaires, habitants de différents quartiers et issus de différentes classes sociales? La lecture de la presse locale et nationale enrichie l'analyse. Les entretiens ont été conduits en langue turque et traduits par mes soins, tout comme les autres matériaux collectés.

\section{PRODUCTION D'UNE SOCIÉTÉ DISCIPLINÉE ET CRIMINALISATION DES PRATIQUES DISSIDENTES}

Le modèle AKP inclut une redéfinition de l'urbanité et de la citoyenneté ("vatandaş $l k »$ ) ainsi qu’une incrimination de la demande sociale sur des logiques partisanes. La production de lieux, de comportements et la redéfinition exclusive de la citoyenneté à partir d'un registre répressif envers tout comportement dissident participent à renforcer la frontière entre les insiders et les outsiders à la communauté politique. En 2006, cette frontière est objectivée et les actes politiques dissidents sont qualifiés d'actes " terroristes " suite aux nouveaux amendements législatifs de la loi relative à la lutte antiterrorisme de 1991.

5. Les terrains à Mersin ont reçu un financement du Conseil européen de recherche dans le cadre du programme de recherche et d'innovation de l'UE Horizon 2020 (ERC CIVILWARS n669690).

6. Parti kurdiste, branche régionale du parti démocratique des peuples (HDP - Halkların Demokratik Partisi). J'utilise le terme " kurdiste » et non " pro-kurde " afin d'intégrer un ensemble d'acteurs au-delà de leur identité ethnique, qui portent des combats tels les droits culturels et politiques des Kurdes en Turquie (Dorronsoro, Watts, 2009).

7. Les enquêtés sont anonymisés et les noms et détails des institutions ou des entreprises qu’ils représentent ne sont pas communiqués dans ce chapitre pour des raisons de sécurité. 
Le projet de l'AKP est à la fois producteur et transformateur de lieux : il produit de nouveaux espaces et rapports à la ville. Les " dispositifs spatiaux " ${ }^{8}$, autrement dit les infrastructures - centres commerciaux, mosquées, logements - sont des opérateurs de traduction du projet de l'AKP. Ils transforment et mettent en scène les intérêts du groupe dirigeant. Ils sont également des supports de délégation et de diffusion de valeurs qui contribuent à la réalisation du projet et à la définition de nouvelles normes dans l'espace social. Alors que les cafés sont des hauts lieux historiques de l'activité partisane en Turquie (Gourisse, 2014), l'AKP promeut une vision de la sociabilité turque qui met les centres commerciaux au cœur des interactions quotidiennes (Débarre, Pérouse, 2016; Pérouse, 2017). Les malls sont désormais, en Turquie, des « espaces naturels de la vie sociale " (ibid.), des lieux de socialisation qui promeuvent des valeurs communes consuméristes. Laccès et la fréquentation des centres commerciaux sont surveillés par la police et par des entreprises de sécurité privées, les clients sont ainsi soumis à certaines formes de " contraintes situationnelles " (Goffman, 2013). Le réaménagement urbain et la politique de logement participent à l'homogénéisation et au gommage des logiques de classe ou identitaires (politiques, ethniques, sociales) dissidentes, au profit d'une identité consumériste?

Cette production de lieux s'accompagne d'une production de comportements. L'AKP façonne les habitus (Doğan, 2016). Sevinç Doğan décrit le parti comme un " champ " (" alan »), au sens bourdieusien, intermédiaire entre le monde social et l'État (2016, p. 14). Pour le responsable du centre d'éducation du peuple à Mersin, une institution directement reliée au ministère de l'Éducation nationale, son institution fait office de "station intermédiaire » (" ara istasyon ») qui a pour but de " camoufler " une autre forme de culture et "d'urbaniser " la population. À la question "que veut dire 'urbaniser' ? ", le fonctionnaire répond que l'urbanisation est visible à partir de quatre critères : " les vêtements, la langue, le travail et la familiarisation (tanıma) ». Il explique que le terme de "familiarisation » renvoie notamment à la fréquentation des lieux de consommation de masse : «ils [les migrants venus de l'Est et du Sud-Est issus de l'exode rural ou politique] sont allés à Forum [grand complexe commercial qui se développe dans plus en plus de villes]. Ils ont vu les magasins, la richesse. Ils ont vu un monde très riche. Ils ont vu des gens très différents s'habiller différemment. Ils sont allés au cinéma. Ils ont vu l'urbanisation $»^{10}$. Ses propos renvoient à la définition d'une identité consumériste développée en accord avec le modèle AKP. Les enseignants des centres vont directement dans les lieux d'habitation pour proposer une forme de rééducation à la citoyenneté.

La redéfinition du milieu urbain s’accompagne également d'une redéfinition de la citoyenneté en tant que communauté politique partisane considérée comme un dispositif répressif, car exclusif et punitif. Ce processus de définition du «bon »

8. Sur la notion de « dispositif spatial » voir Lussault (2007).

9. Ceci passe notamment par la destruction de quartiers contestataires et leurs reconstructions selon les normes désirées par l'AKP à l'aide de l'Administration du Logement collectif, TOKI (Toplu Konut İdaresi Başkanliğ $r)$ (Pérouse, 2013).

10. Entretien avec le responsable du Bureau des projets de Recherche-Développement de la ville de Mersin, à Mersin, le 25 avril 2018. 
et du « mauvais » citoyen, comme cela a déjà été interprété dans le cas de la Tunisie sous le régime de Ben Ali, peut être analysé comme une technique disciplinaire (Hibou, 2005). À l'occasion de la « $23^{\mathrm{e}}$ réunion des mubtars» (les mubtars sont les chefs de quartiers élus au suffrage universel direct par les habitants du quartier) du 6 avril 2016 et lors de la cérémonie $563^{\mathrm{e}}$ anniversaire de la conquête de Constantinople [Istanbul] le 26 mai 2016 à Yenikapr, Erdoğan décrit une nouvelle forme de citoyenneté exclusive, qui peut être retirée à ceux qui sont considérés comme " terroristes » (Öney, 2016). La redéfinition de la citoyenneté par l'État-AKP défini l'outsider avant de créer un "nous" (ibid.) et s'inscrit dans un mécanisme de répression unilatérale. Ce discours s'inscrit dans la lignée de celui prononcé en mars 2005 par le chef d'État-major des armées suite à l'évènement du prétendu drapeau brûlé à Mersin lors des célébrations du Newroz, Nouvel An kurde, dans lequel les auteurs sont désignés comme des "soi-disant citoyens " ( sözde vatandaş") (Gambetti, 2013). Pour Zeynep Gambetti, ce discours participe à brouiller les frontières entre les sympathisants du PKK et les non-sympathisants ainsi que tous ceux qui ne soutiendraient pas le discours nationaliste turc tel qu'il est défini par l'État (ibid.).

Au regard de la loi, cette redéfinition de la citoyenneté saccompagne de la redéfinition du " non-citoyen » à partir de l'élargissement de la catégorie de « terroriste ". La fabrique d'un " ennemi intérieur " permet à l'État de développer des technologies de pouvoir coercitives au nom de la sécurité et de renforcer sa formation identitaire nationaliste (Huysmans, 1998). En 2006, les amendements apportés à la loi antiterroriste no 3713 de 1991 [Terörle Mücadele Kanunu] (qui s’aligne sur les tendances globalisées post-2001) étendent la liste des actes considérés " terroristes ». Ainsi, sous l'AKP, cette définition intègre toute activité dissidente et institue une forme de violence légale (Gambetti, 2013 ; Yonucu, 2017). Les révisions de la loi de 1991 visent dans un premier temps les activités dissidentes entreprises par les militants kurdes et alévis qui sont perçues comme portant atteinte à la souveraineté de l'État, puis s'exportent à la classe moyenne et supérieure sunnite (ibid.). «L'ennemi intérieur » prend la forme de «terroristes » : ils « se dissimulent, se fondent dans la normalité de la coexistence quotidienne des gens, sans se donner à voir " (Linhardt, 2001, p. 77). "L'ennemi " peut être partout, et la menace prend alors un caractère diffus au sein de la société. Début 2017, dans le contexte post tentative de coup d'État, la publication d'un décret permet de priver les citoyens turcs, vivant en dehors du pays et accusés de "terrorisme ", de leur nationalité. ${ }^{11}$.

L'incrimination de la demande sociale à partir du registre " terroriste " est effectuée par les élites dirigeantes AKP en cas d'opposition aux politiques gouvernementales. Dans la ville de Hopa le 31 mai 2011, le jour de la visite d'Erdoğan, des manifestations sont organisées par le premier parti d'opposition, le CHP, le parti kurdiste BDP et des militants de l'organisation de la Halkevi ${ }^{12}$, pour

11. Un rapport a été publié à ce sujet : «Arbitrary deprivation of nationality and denial of consular services to Turkish citizens ", Institute on Statelessness and Inclusion, July 2017. Disponible en ligne: https://files.institutesi.org/policy-brief-Turkey-arbitrary-deprivation-of-nationality_2017.pdf.

12. Halkevleri est un mouvement politique de tendance socialiste. 
s'opposer à la privatisation de la production d'électricité. Les manifestants sont violemment réprimés par la police. Erdoğan les accuse d'être affiliés au Parti des Travailleurs du Kurdistan (PKK). Ils sont poursuivis en justice pour " appartenance à une organisation illégale " (Gambetti, 2013, p. 143).

Laccès ou l'interdiction des aides sociales sont laissés à l'appréciation des conduites des individus par les dirigeants. Lobtention de la carte de soutien (destek kart - carte prépayée rechargeable permettant de faire ses courses dans les marchés sélectionnés par le pouvoir local) est conditionnée à une logique partisane de "récompense-punition " $:$ si les conduites ne correspondent pas aux valeurs du parti, le processus d'accès à la carte de soutien peut alors être enrayé, voire empêché $(A r k, 2015)^{13}$. Les aides sociales acquises peuvent être retirées conséquemment à un comportement perçu comme dissident. À Adana en octobre 2008, des manifestations ont lieu pour protester contre le mauvais traitement présumé du leader du PKK. Les familles responsables des 325 individus mineurs participants sont menacées de perdre la gratuité des soins médicaux par le biais de la suppression de la " carte verte " ("yeşil kart») une action en justice sur motif que les " terroristes " ne devraient pas détenir cette carte, et demande une suppression des aides au charbon données par la Fondation d'assistance sociale et de solidarité liée à l'État (Sosyal Yardimlaşma ve Dayanışma Vakfi) pour chauffer les foyers ${ }^{15}$. Le gouverneur ne considère pas ces mesures comme étant "une menace ou une tentative d'intimidation ". Au contraire, il souligne sa volonté de protéger les enfants, action légitime au regard de la loi, et appelle les familles à en faire de même ${ }^{16}$. Au cours de ces manifestations, plusieurs enfants auraient été torturés par la police, et certains sont morts noyés dans un canal. Pour İlhan Atış, les familles des enfants morts doivent également être concernées par ces mesures ${ }^{17}$. Bien que celles-ci ne soient pas soutenues par le ministre de l'Éducation nationale ${ }^{18}$, l'incident entraînera tout de même une réévaluation des 514000 dossiers des détenteurs des cartes vertes et l'annonce en janvier 2009 d'une radiation de 30 à 40000 bénéficiaires de l'aide sociale ${ }^{19}$.

La production de lieux et de comportements s'effectue parallèlement à l'incrimination des protestations et à la construction d'une menace " terroriste ». Les

13. Par ailleurs, cette politisation de l'aide sociale, et le risque d'un non-accès à cette dernière, entravent les comportements contestataires à l'échelle des quartiers, notamment les mobilisations contre les projets fonciers tels que ceux de régénérations urbaines dont nous avons évoqué les logiques en amont (Ark, 2015).

14. La " carte verte » est un document remis aux personnes qui ne possèdent pas la sécurité sociale du fait d'un emploi informel ou de faibles revenus et leur permet d'accéder aux services de santé gratuitement.

15. « Adana Valisi Atış่tan çarpıcı açılamalar », Hürriyet.com.tr, 4 novembre 2008 : https://www. hurriyet.com.tr/gundem/adana-valisi-atistan-carpici-aciklamalar-10282650.

16. " «Ya eylem ya yeşil kart» tavrı destek bulmadı », CNNTürk.com, 31 octobre 2010, https:// www.cnnturk.com/2008/turkiye/10/31/ya.eylem.ya.yesil.kart.tavri.destek.bulmadi/498815.0/index.html.

17. «Eylemci çocukların ailelerine yeşil kart cezası », t24.com, 29 octobre 2008 : https://t24.com.tr/ haber/eylemci-cocuklarin-ailelerine-yesil-kart-cezasi,13768.

18. CNNTürk.com, op. cit., 2010.

19. «Adana’da 40 bin yeşil kart iptal edilecek », İHA.com.tr, 29 janvier 2009 : https://www.iha.com. tr/haber-adanada-40-bin-yesil-kart-iptal-edilecek-53418/. 
changements législatifs correspondent à un mode d'objectivation de cette menace paradoxalement diffuse. Dans une société verrouillée par des logiques partisanes conditionnant l'accès aux ressources étatiques, les individus sont amenés à s'investir dans un conflit polarisé qui traverse la société turque entre les défenseurs du modèle AKP et leurs opposants. L'État-parti délègue son monopole de la violence légitime aux civils et aux acteurs privés tout en contrôlant et en arbitrant les mécanismes coercitifs développés.

\section{DÉLÉGUER ET LÉGITIMER LES PRATIQUES COERCITIVES CIVILES ET PRIVÉES}

Au sein de ce modèle, qui définit à la fois l'ordre social promu par l'AKP et exclut toute forme dissidente, une partie de la population participe à la société de surveillance et devient partie prenante de l'entretien des dispositifs répressifs ${ }^{20}$. Sous l'AKP, on observe des dynamiques de "laisser-faire "21, d'encouragement voire de légalisation des pratiques coercitives civiles sous forme de lynchages ou de pratiques qui oscillent entre "dénonciation " et " délation $~^{22}$. Cette forme de privatisation de la répression s'ajoute au rapprochement entre les entreprises privées de sécurité et l'État.

Les pratiques coercitives perpétrées par les civils ne sont pas inhérentes à la période AKP, celles-ci sont également présentes dans l'Empire ottoman (Aykut, 2011) et sont intégrées au processus de construction nationaliste contre ceux qui sont perçus comme une menace à la souveraineté de l'Empire puis de l'État (Bora, Yonucu, 2019). Au cours de la période républicaine, outre les groupes perçus comme une menace par les élites dirigeantes, la population dans son ensemble peut également être perçue comme telle ${ }^{23}$. Dans les années 1980, la population est armée par l'État et est directement investie dans le conflit entre l'armée et le PKK. Plus récemment, Erdoğan appelle les citoyens à la violence lors de son appel FaceTime dans la nuit du 15 au 16 juillet 2016 diffusé en direct dans les médias. Le président encourage les citoyens à descendre dans la rue pour s'opposer aux militaires putschistes ${ }^{24}$.

Les pratiques de lynchages peuvent être motivées par des logiques morales ou être de l'ordre d'un « réflexe national " (Bora, 2008) Dans certains cas, des individus décident par eux-mêmes de faire la loi au sein d'un espace précis, le quartier,

20. L'intégration de la population aux mécanismes de surveillance comme mode de domination peut être analysée à partir d'une lecture des processus développés par la Stasi en RDA (Combe, 1999).

21. Le " laisser-faire " peut devenir " un mode de gouvernement qui, dès lors, peut aussi se révéler être une technique de discipline et de surveillance en permettant, dans ces circonstances extrêmement ponctuelles et circonscrites, de punir et de désigner les "ennemis"» (Hibou, 2011, p. 214).

22. Les termes « délation" ou "dénonciation " sont définis à partir des propos de Luc Boltanski (1984) : la délation serait la " dénonciation d'un individu " tandis que la dénonciation serait la " dénonciation d'une injustice », qui pourrait prendre la forme en Turquie d'une protestation sociale et politique ayant pour objet la défense du modèle AKP.

23. Pour Mustafa İsmet Inönü, le second président de la République de Turquie, la nation entière était considérée comme un ennemi (Bora, Yonucu, 2019, p.229).

24. «Erdoğan calls his people», Yeni Şafak, Youtube, 5 juin 2017. 
afin de contrôler des comportements perçus comme déviants à partir d'un registre basé sur la moralité, telle la consommation d'alcool aux abords d'une mosquée (Massicard, 2019)25. Dans d'autres, des actes de lynchages isolés ou la mobilisation d'un " réflexe national " peuvent déclencher des vagues de violences collectives à travers plusieurs villes du pays. En réaction au prétendu drapeau brûlé à Mersin en 2005, à Trabzon, une foule attaque dans la rue cinq adhérents en train de tracter pour l'association kurdiste de solidarité et d'entraide des proches de condamnés et de détenus (TUYAD, Tutuklu ve Hükümlü Yakınları Yardımlaşma ve Dayanışma Derneği $i$. Les assaillants scandent des slogans rappelant qu'« ici ce n'est pas Mersin ", ou bien "nous allons brûler ceux qui osent brûler le drapeau turc ». Par la suite, entre 2005 et 2013, plus de 130 cas de lynchage et de violences collectives sont recensés (Gambetti, 2013, p. 126). Lutilisation du terme « réflexe national », bien qu'ancré historiquement dans l'univers politique turc de l'extrême droite au sein du MHP (Bora, 2008) - allié à l'AKP depuis 2018 - permet d'insister sur le caractère " non contestable » de tout comportement défendant la nation turque puisque ceux-ci sont tolérés par le pouvoir.

En effet, le degré de répression des actes de lynchages est aussi défini selon une logique partisane. Le pouvoir peut réprimer les initiateurs ou les victimes si ceuxci s'opposent au modèle de société partisane développé par l'AKP. À l'inverse, il peut encourager ou " laisser-faire » des violences qui défendent ce même modèle. Les cinq individus de la TUYAD ont été condamnés à 2 à 4,5 ans de prison pour " indignation sociale " alors que les auteurs des lynchages ont été condamnés à 2 à 6 mois de prison (Gambetti, 2007, p. 14). Autre exemple, des barrages routiers sont établis par des groupes d'individus sur la départementale D-400 entre Antalya et Mersin en septembre 2015 afin de stopper des bus collectifs identifiés comme « kurdes ». Cet événement succède aux attaques du PKK dans la province d'Hakkari tuant une dizaine de soldats de l'armée turque et de policiers dans la province d'Iğdır. Les Fondations des Foyers idéalistes pour la culture et l'éduca-

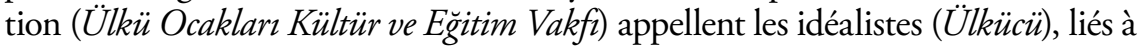
l'extrême droite, à descendre dans la rue pour faire valoir le « Respect du martyr, la malédiction de la terreur " ("Şehide Sayg Teröre Lanet ") ${ }^{26}$. Les bus pris pour cible (pneus crevés, vitres cassées, jets de pierres) sont identifiés à l’aide du nom de l'entreprise qu'ils portent, renvoyant à un ancrage territorial dans les régions kurdes de Turquie : "Diyarbakır Seyahat », «Öz Diyarbakır », "Star Diyarbakır» et "Van Gölï Seyahat" et "Mardin Seyahat "27. La presse relate que certains chauffeurs ont été arrêtés par la police (Çelik et al., 2015). Un habitant de Mersin ajoute que la police n'aurait rien fait pour empêcher les groupes bloquant la route lors d'un événement similaire ${ }^{28}$. À partir de ce type d'exemples, Zeynep Gambetti

25. Ces pratiques ne sont pas inhérentes à la période $\mathrm{AKP}$ et possèdent une complexité historique qui leur est propre (Massicard, 2019).

26. « Devlet Bahçeli Ülkücüleri sokağa gönderiyor! », Sabah.com, 9 septembre 2015 https://www.sabah.com.tr/gundem/2015/09/08/devlet-bahceli-ulkuculeri-sokaga-gonderiyor.

27. Entretien avec un entrepreneur en bâtiment à Mersin, le 16 juin 2018. Il n’a pas pu aller travailler ce jour-là à cause des évènements, son chantier étant situé dans la zone et la route étant fermée. Information confirmée dans la presse, voir Çelik B. et al. (2015).

28. Idem. 
observe une forme de "vigilantisme légal " (legal vigilantism) (Gambetti, 2013, p. 138) et une reconstruction de l'État au sein de la société : «the "people" act as if they are the state : they appropriate and resignify the state function of dispensing justice " (ibid., p. 140). Sous l'AKP, il existe donc une forme « d'appropriation privée de la justice et de la violence» (Das, Poole, 2004 cité par Gambetti, 2013, p. 140). De plus, j'observe la constitution d'une forme de "société civile " ${ }^{29}$ étatisée et au service de l'État-parti.

Les pratiques de dénonciation et de délation sont à ajouter aux pratiques de lynchages et à intégrer aux modalités de surveillance des espaces privés comme mode de contrôle social. Elles peuvent être encouragées par des acteurs privés, mais aussi par l'État, et parfois détournées par des acteurs civils qui cherchent à en tirer profit. À Diyarbakır, le gouvernement délègue les pratiques de surveillance des comportements dissidents, comme la fraude à la consommation, à l'entreprise privée de distribution d'électricité, qui possède des liens étroits avec l'AKP (Pineau, 2015). Dans un contexte d'hybridité des relations public-privé et de privatisation des services public ${ }^{30}$, l'entreprise privée apparait comme site de domination et de gouvernement à distance pour l'État. La société privée de distribution de l'électricité, DEDAŞ, encourage la dénonciation d'un voisin qui consommerait illégalement de l'électricité. Un numéro de téléphone ou encore une page spéciale sur le site internet de la compagnie intitulée " notification d'utilisation illégale " ("kaçak kullanım bildirimi ») sont mis en place ${ }^{31}$. L'État investit la sphère privée des individus et intègre dans ses pratiques gouvernementales la dimension conflictuelle des rapports entre les individus comme dans le cas de la Stasi en RDA étudiée par Sonia Combe (Combe, 2005 ; Hibou, 2011). La pratique de la dénonciation est renforcée par le déplacement des compteurs électriques. L'entreprise privée les déplace de l'intérieur des appartements au rez-de-chaussée dans le hall collectif ${ }^{32}$. Il est alors plus difficile pour les consommateurs de falsifier leur compteur en étant exposé aux regards des voisins à l'entrée de l'immeuble.

L'État peut directement encourager et récompenser les pratiques de délation entre les citoyens. Le 31 août 2015, une loi publiée au Journal officiel par le ministère de l'Intérieur réglemente les récompenses de " ceux qui ont aidé les auteurs de crimes en vertu de la loi antiterroriste " en fixant le montant maximum à 200000 TL $(20000 €)$, montant pouvant être multiplié jusqu’à 20 fois sous

29. En Turquie la catégorie « société civile » peut-être utilisée par certains acteurs turcs urbains « qui se dissocient de plus en plus des réseaux politiciens traditionnels » (Groc, 1998, p. 2) et cherchent à autonomiser le social du politique tout en posant un regard critique d'un État qui ne serait pas capable de gérer la diversité de la société turque et qu'il chercherait à homogénéiser (ibid.). Dans les pratiques de lynchage présentes, on observe une forme de " société civile " pro-AKP.

30. Les privatisations représentent 8,2 milliards de dollars entre 1985 et 2003; elles sont passées à 50,3 milliards de dollars sous l'AKP entre 2004 et 2013 (Massicard, 2014). Concernant les l'hybridité des relations public-privé en Turquie voir Pérouse (2013).

31. Une page spéciale consacrée à cet usage est disponible sur le site de la compagnie d'électricité : https://www.dedas.com.tr/kacakkullanimbildirimi.aspx.

32. Entretien avec le responsable de l'entreprise privée de distribution d'électricité, DEDAŞ, à Diyarbakır, le 24 avril 2015. 
certaines conditions ${ }^{33}$. Cette loi est établie pour encadrer la mise en application, le 30 octobre 2015 selon la Direction des Affaires religieuses, de la hotline "Alo 140 terör ihbar hattı ", un numéro d'urgence de dénonciation du terrorisme ${ }^{34}$. Une vidéo réalisée par le département antiterroriste de la Direction générale de la sûreté, acceptée comme spot "d'intérêt public " lors de la réunion du Conseil suprême de la radio et de la télévision le 7 septembre 2017, est diffusée afin de solliciter la population : "nous pouvons arrêter le terrorisme ensemble " (" terörü hep birlikte durdurabiliriz ») ${ }^{35}$. Pour Gülşen Özbek, avocate au sein de l'association des avocats pour la paix, ÖHP (Özgürlükçü Hukukçular Platformu), ce dispositif participe à la polarisation de la société et à l'accroissement d'un sentiment d'insécurité (Berk, 2018). Sur le site internet du ministère de l'Intérieur, cinq listes d'individus recherchés sont accessibles. Le montant de la récompense varie selon la couleur de la liste : de 10000000 TL (1 $000000 €)$ pour la liste rouge à 500 000 TL (50 $000 €$ ) pour la liste grise ${ }^{36}$. Les pratiques de dénonciation du terrorisme par téléphone ont été détournées, dans un but lucratif, par des hackers non identifiés. Des SMS sont envoyés sur les numéros turcs. L'envoyeur, indiqué sur l'écran comme étant la " police " ("polis»), un " procureur» ("savcı») ou encore "l'armée » (" asker»), invite au versement d'argent en faveur de la lutte antiterroriste. Sur certains SMS, il accuse les citoyens destinataires de ces messages d'être liés aux organisations " terroristes » de $\mathrm{FETÖ}^{37}$, du PKK et de DAEŞ et réclame de l'argent. La police lance une campagne de sensibilisation contre les fraudes, pour prévenir les citoyens. Ils sont encouragés à dénoncer ces pratiques via le numéro d'urgence de la police secours « 155 ».

Enfin, j’observe également une forme de privatisation de la sécurité. Les services de sécurité étatiques chapeautent plusieurs entreprises privées du secteur. Selon un rapport d'activités de la Direction générale de la police, en 2009, 513134 gardes de sécurité sont entraînés et certifiés par la Direction générale de la sûreté (Taşdelen et al., 2010, p. 91 cité par Bozbeyoğlu, 2017, p. 147). Également, les filles du ministre des Finances, Naci Ağbal ${ }^{38}$, auraient intégré à l'entreprise familiale des activités liées au développement de divers types de système de surveillance (ibid. p. 147).

33. «Le montant de la récompense peut être augmenté jusqu’à 20 fois avec la recommandation de la commission et l'approbation du ministre de l'Intérieur si le contrevenant est un cadre supérieur de l'organisation terroriste ou selon l'indignation publique suscitée par le crime " : «'Alo 140' terör ihbar hatt1 ", Haber7.com, 24 octobre 2015 https://www.haber7.com/guncel/haber/1620090-alo140-teror-ihbar-hatti-kuruldu

34. Louverture de cette ligne est justifiée par l'augmentation des « actes terroristes » au cours de cette période qui correspond à la reprise des combats entre l'armée turque et le PKK suite à la reconduction des élections législatives du 7 juin 2015 où l'AKP perd la majorité au parlement et où le parti kurdiste franchi le barrage des $10 \%$ qui lui permet pour la première fois de siéger au Parlement. 35. Information disponible sur le site internet du ministère des Affaires religieuses : https://ankara. diyanet.gov.tr/bala/sayfalar/contentdetail.aspx?ContentId=264\&MenuCategory=Kurumsal.

36. Information disponible sur le site internet du ministère de l'Intérieur : http://www.terorarananlar.pol.tr.

37. FETÖ désigne l'organisation du mouvement Gülen accusé par l'État-parti d'avoir organisé la tentative de coup d'État du 15 juillet 2016.

38. Présumé en poste au moment de la publication de cette information par Alanur Çavlin Bozbeyoğlu (2017). 
Sous l'AKP, la définition de l'ennemi intérieur s'étend à toute conduite et pratique dissidente face aux politiques gouvernementales et la répression n'est plus limitée aux groupes perçus comme séparatistes. J'observe l'importance de l'usage des conflits, parfois suscités ou amplifiés au gré des opportunités circonstancielles, et l'exercice de la délation comme technique de gouvernement qui permet un contrôle social de la dissidence et une affirmation du modèle AKP. Finalement, en Turquie, la délation encouragée par l'État sous ses différentes formes pose la question suivante : « la délation peut-elle être civique ? (Jobard, Brodeur, 2005, p. 208).

\section{CONCLUSION}

Sous l'AKP s'opère une transformation des techniques de gouvernement multipliant des dispositifs répressifs qui auraient pour fonction de pallier à une insécurité croissante, sans pour autant mettre en doute les effets de ces dispositifs sur la construction sociale du crime ou de la " menace » et la catégorisation de faits comme problèmes (Bigo, 2001). Néanmoins, la construction globalisée de la menace terroriste dans un contexte post-2001 est utilisée en Turquie comme une modalité de contrôle social des comportements dissidents. La définition du "bon " et du " mauvais » citoyen répond à une logique partisane de "récompensepunition " plus large qui dirige l'ensemble des champs de la société. Les subjectivités dominées par le pouvoir sont façonnées à l'image des villes que l'ÉtatAKP investit par des valeurs consuméristes qui tendent à gommer les identités ethniques et religieuses et les lieux contestataires au profit d'une identité partisane. Tout comme l'aide sociale est conditionnée au soutien au parti, la criminalisation des conduites s'appuie sur des logiques partisanes. Un conflit entre deux individus (cf. les cas de délation) ou deux groupes d'individus (cf. les cas de lynchage) peut être légitimé par l'État si les initiateurs sont considérés par ce dernier comme des avocats de la défense du modèle. Le conflit peut également être encouragé par l'État ou les entreprises privées, liées à un État-parti qui conditionne l'accès au champ économique (Esen, Gümüşçü, 2018), à travers des outils (cf. hotline) développés à cet effet. À l'inverse, des conduites qui s'opposent aux politiques gouvernementales ou dérogent à la citoyenneté $\mathrm{AKP}$ sont fortement réprimées à partir du registre de la menace « terroriste ». Les changements législatifs viennent objectiver cette menace qui reste paradoxalement diffuse au sein de la société. Ce paradoxe participe à créer un climat d'incertitude et de peur au sein de la population qui renforce la légitimité du discours sécuritaire prodigué par l'État et légitimé par le contexte mondial. Ce mécanisme qui s’auto renforce est également visible à l'échelle régionale, dans le cas du conflit syrien, où l'AKP utilise un procédé discursif similaire afin de légitimer une politique d'ingérence et obtenir le soutien des pays occidentaux (Sevim, 2017).

À l'échelle nationale, l'installation et l'entretien de mécanismes disciplinaires par l'État-parti et par la population sont à relier au développement d'outils 
légitimés par les organisations internationales. L'AKP entreprend une « informatisation du gouvernement " (Bonditti, 2013) qui permet à l'Etat d'augmenter significativement les pouvoirs donnés au traditionnel « œil de l'État », l'administration, tout en faisant appel à la participation des citoyens afin de mieux connaître et de mieux contrôler la société (Jobard, Brodeur, 2005, p. 199). Dans le cadre du développement de l' " $e$-Devlet » (e-État), élaboré suite à la participation de la Turquie à la conférence e-Europe en juin 2001 organisée par l'Union européenne, et qui s'inscrit dans «l'objectif Turquie digitale! " ( Dijital Türkiye hedefi!»), l'État-AKP met en place la base de données d'enregistrement civil MERNİS (Merkezi Nüfus İdaresi Sistemi), financée par un prêt d'aide à la mise en œuvre de la privatisation (PIAL) de la Banque mondiale. Depuis 2003, les informations sur les citoyens sont peu à peu centralisées sur cette base. Un simple accès au dossier lié au numéro unique de citoyen dans la base de données MERNIS permet de connaître les antécédents de santé, prêts ou interdits bancaires, le solde des comptes bancaires, les diplômes obtenus, l'état du casier judiciaire, etc. (Avşar, 2017) ${ }^{39}$. La centralisation et l'utilisation des données collectées par l'État, justifiées par le renforcement de la performance bureaucratique et des services, seraient paradoxalement « une sorte de masque démocratique de la domination» (Topal, 2005, p. 90). De plus, en posant l'argument que les usages de la technologie sont dépendants de leurs usages sociau $\mathrm{x}^{40}$, ces outils apparaissent comme des dispositifs de surveillance, où les individus en y participant sont à la fois cibles, acteurs et instruments ${ }^{41}$.

39. MERNIS est initialement pensée dans les années 1970 comme un registre électronique de la population, l'idée de départ est étendue à l'intégration jusqu'à aujourd'hui d'une cinquantaine de sous-projets tels que la création d'un numéro d'identification unique pour chaque individu qui est utilisé dans divers administrations et services (Avsar, 2017).

40. Notes issues d'une conférence donnée par Didier Bigo, intitulée « Le renseignement à l'épreuve des données numériques. Usages, coopérations, légitimités ", dans le cadre du séminaire organisé par l'équipe de l'ERC «Social Dynamicsof Civil Wars», Paris 1 Panthéon Sorbonne, le 20 novembre 2019.

41. Je remercie Işıl Erdinç pour son accompagnement et ses précieuses suggestions, Denia Chebli pour ses remarques constructives et sa relecture attentive ainsi qu'Adam Baczko et Gilles Dorronsoro pour nos discussions stimulantes. 


\section{RÉFÉRENCES / REFERENCES}

Ark C. (2015), Transformation urbaine et réseaux clientélistes, le quartier de Sabintepe à Istanbul, Thèse de doctorat en science politique, Paris 1 Panthéon Sorbonne.

Ark C., Smyrl M. (2017), «Innovation ouverte et "Living Labs" : production et traduction d'un modèle européen ", Revue française d'administration publique, ENA, n 161, p. $89-102$.

Avşar S. A. (2017), " “Congratulations, your daughter is pregnant!": right to privacy in Turkey", Research and Policy on Turkey, vol. 2, n' 1, p. 38-48.

Aykut E. (2011), "Ethnic conflict, the Armenan question, and mob violence in the late Ottoman empire ", in Berg M., Wendt S. (eds.), Globalizing Lynching History. Vigilantism and extralegal punishment from an international perspective, New York, Palgrave Macmillan, p. 119-135.

Bayart J.-F. (2008), « Hégémonie et coercition en Afrique subsaharienne. La "politique de la chicotte" ", Politique africaine, ${ }^{\circ} 110$, p. 123-152.

Berk S. (2018), "İhbar hattı uygulaması toplumda güvensizliği artırır », evrensel.net, 18 janvier 2018.

Bigo D. (1998), « Sécurité et immigration : vers une gouvernementalité par l'inquiétude? ", Cultures et Conflits, $\mathrm{n}^{\circ}$ 31-32, p. 13-38.

Bigo D. (2001), « Internal and External Security(ies): The Möbius Ribbon » in Albert M. (ed.), Identities, Borders, Orders, University of Minnesota Press, p. 91-116.

Boltanski L., Darré Y., Schiltz M.A (1984), "La dénonciation ", Actes de la recherche en sciences sociales, vol. 51, p. 3-40.

Bonditti P. (2013), " (Anti) terrorisme. Mutations des appareils de sécurité et figure de l'ennemi aux États-Unis depuis 1945 », Critique internationale, n 61, p. 147-168.

Bora T. (2008), Turkiyénin Linç Rejimi, Istanbul, Birikim Yayinlari.

Bora T., Yonucu D. (2019), "State and Civilian Violence Against "Dangerous" Others Tanıl Bora and interviewed by Deniz Yonucu ",in Özyürek E., Özpınar G., Altındiş E. (eds.), Authoritarianism and Resistance in Turkey: Conversations on Democratic and Social Challenges, Boston, Springer, p. 229-235.

Bozbeyoğlu A. C. (2012), "The electronic eye of the police: the provincial information and security system in Istanbul ", in Doyel A., Lippert R., Lyon D. (eds.), Eyes everywhere, the global growth of camera surveillance, London and New York, Routledge.

Çelik B., Çetin R., Gümüş Ç., Özcan Ş. (2015), « Türkiye’nin alacakaranığı », BasHaber, 14-20 septembre.

Combe S. (2005), " La Stasi ", in Brodeur J.-P, Jobard F. (dir.), Citoyens et Délateurs. La délation peut-elle être civique?, Paris, Autrement.

Combe S. (1999), Une société sous surveillance. Les intellectuels de la Stasi, Paris, Bibliothèque Albin Michel, coll. «Idées ».

Débarre S., Pérouse J.-F., (2016), « Dire et faire le territoire en Turquie : entre idéologie unitariste et obsession de la rente ", European Journal of Turkish Studies, n 23.

Doğan S. (2016), Mahalledeki AKP: Parti İsleyiz̧i, Taban Mobilizasyonu ve Siyasal Yabancılaşma [L'AKP dans le quartier : fonctionnement du parti, mobilisation de la base et aliénation politique], Istanbul, İletişim Yayınları.

Dorronsoro G. (2017), «L'État-AKP et le gouvernement par la crise », Mouvements, $\mathrm{n}^{\circ}$ 90, p. 11-18.

Dorronsoro G., Watts N. (2009), "Toward Kurdish Distinctiveness in Electoral Politics », International Journal of Middle East Studies, vol. 41, n 3, p. 457-478. 
Esen B., Gümüşçü S. (2018), « Building a Competitive Authoritarian Regime: StateBusiness Relations in the AKP's Turkey ", Journal of Balkan and Near Eastern Studies, vol. 20, n ${ }^{\circ}$, p. 349-272.

Foucault M. (2004), Sécurité, Territoire, Population, cours au Collège de France. 1977-1978, Paris, Gallimard, Seuil.

Gambetti Z. (2013), " "I'm no terrorist, I'm a Kurd": Societal Violence, the State and Neoliberal order » in Gambetti Zeynp, Godoy-Anativia Marcial (eds.), Rhetorics of Insecurity. Belonging and violence in the neoliberal era, Social Science Research Council, New York University Press, p. 125-152.

Gambetti Z. (2007), " Linç girişimleri, neoliberalizm ve güvenlik devleti », Toplum ve Bilim, $\mathrm{n}^{\circ} 109$, p. 7-34.

Goffman E. (2013), Comment se conduire dans les lieux publics? Notes sur l'organisation sociale des rassemblements, Economica, traduit par D. Cefaï, Economica.

Gourisse B. (2014), La violence politique en Turquie. L'État en jeu (1975-1980), Paris, Karthala, collection "Recherches internationales".

Groc G. (1998), "La "société civile” turque entre politique et individu », Cahiers d'Études sur la Méditerranée Orientale et le monde turco-iranien, n 26, p. 43-74.

Güler A., Kula S. (2016), « Smart public safety: application of Mobile Electronic System Integration (MOBESE) in Istanbul ", in Gil-Garcia J.R., Pardo T.A., Nam T. (eds.), Smarter as the New Urban Agenda A Comprehensive View of the 21st Century City, Public Administration and Information Technology, $n^{\circ} 11$, p. 243-258.

Hibou B. (2011), Anatomie Politique de la Domination, Paris, La Découverte.

Hibou B. (2005), "Économie politique de la répression : le cas de la Tunisie ", Raisons politiques, vol. $4, \mathrm{n}^{\circ} 20$, p. 9-36.

Hibou B. (1999), "La "décharge”, nouvel interventionnisme », Politique africaine, vol. 73, $\mathrm{n}^{\circ} 1$, p. 6-15.

Huysmans J. (1998), "Dire et écrire la sécurité : le dilemme normatif des études de sécurité ", Cultures et Conflits, p. 31-32.

Jobard F., Brodeur J-P (2005), "Conclusion : le pouvoir obscur de la délation », Brodeur J.-P, Jobard F. (dir.), Citoyens et Délateurs. La délation peut-elle être civique?, Paris, Autrement.

Linhardt D. (2001), "L'économie du soupçon. Une contribution pragmatique à la sociologie de la menace ", Genèses, n 44, p. 76-98.

Lussault M. (2007), L'Homme spatial: la construction sociale de l'espace humain, Paris, Seuil.

Massicard E. (2019), "Quand les civils maintiennent l'ordre. Configurations vigilantes à Istanbul ", Revue des mondes musulmans et de la Méditerranée, n 145, p. 229-256.

Massicard E. (2014), "A Decade of AKP Power in Turkey: Towards a Reconfiguration of Modes of Government?", Les Études du CERI, n 205, Paris, Sciences Po CERI.

Öney S. (2016), "Üç Boyutlu "Yeni Vatandaşlık” ve Kapsam Dışında Kalanlar », Birikimdergisi.com.

Pamuk O. (2005) [2002], Neige, Paris, Gallimard.

Pérouse J.-F. (2017), Istanbul planète. La ville-monde du XXIe siècle, Paris, La Découverte.

Pérouse J.-F. (2013), "L'État sans le public : quelques conjectures à propos de l'Administration du logement collectif (TOKI) », in Aymes M., Gourisse B., Massicard E. (dir.), L'Art de l'État en Turquie, Arrangements de l'action publique de la fin de l'Empire ottoman à nos jours, Paris, Karthala.

Pérouse J.-F. (2012), " La création de 13 nouvelles municipalités métropolitaines en Turquie par la modification de la loi $\mathrm{n}^{\circ} 5779$ ou le triomphe écrasant de l'urbain dans l'ordre de gestion territoriale ", dipnot.hypothèses.org, 19 novembre. 
Pineau C. (2015, non publié), Privatisation et consommation illégale d'électricité à Diyarbakır : retrait ou redéploiement de l'État ?, Mémoire de recherche en science politique, IEP de Grenoble.

Sevim H. (2017), "Comment (ré)inventer l'“ennemi” ? Le discours des élites politiques turques sur le conflit syrien ", Critique internationale, $\mathrm{n}^{\circ}$ 74, p. 53-68.

Topal Ç. (2005), "Global citizens and local powers: Surveillance in Turkey”, Proof, Duke University Press/Journals, Social Text 83, vol. 23, n 2, p. 85-94.

Yonucu D. (2018), «Urban Vigilantism: A Study of Anti-Terror Law, Politics and Policing in Istanbul ", International Journal of Urban and Regional Research, v.2, n ${ }^{\circ} 3$, p. 408422. 